\title{
On Bootstrap Implementation of Likelihood Ratio Test for a Unit Root
}

\author{
AnTON SKROBOTOV* \\ The Russian Presidential Academy \\ of National Economy and Public Administration
}

February 25, 2018

\begin{abstract}
In this paper we investigate the bootstrap implementation of the likelihood ratio test for a unit root recently proposed by Jansson and Nielsen (2012). We demonstrate that the likelihood ratio test shows poor finite sample properties under strongly autocorrelated errors, i.e. if the autoregressive or moving average roots are close to -1 . The size distortions in these case are more pronounced in comparison to the bootstrap $M$ and ADF tests. We found that the bootstrap version of likelihood ratio test (with autoregressive recolouring) demonstrates better performance than bootstrap $M$ tests. Moreover, the bootstrap likelihood ratio test show better finite sample properties in comparison to the bootstrap ADF in some cases.
\end{abstract}

Key words: likelihood ratio test, unit root test, bootstrap.

JEL: C12, C22.

\section{Introduction}

The implementation of bootstrap in unit root testing has a long history in econometric time series literature. The theory of bootstrap metodology in unit root context was developed in, inter alia, Basawa et al. (1991a,b) and Park (2002, 2003). Chang and Park (2003) considered sievebased implementation of the residual-based bootstrap for standard ADF type test and Park (2006) considered a bootstrap theory for weakly integrated processes. Looking beyond the ADF type test, Cavaliere and Taylor $(2009 a)$ investigated the bootstrap implementation of the so-called $M$ type unit rot tests proposed by Stock (1999). They took attention to the wild bootstrap and heteroskedasticity pattern of underlying errors in their model. It should be noted that Palm et al. (2008) review different bootstrap unit root tests. Smeekes (2012) analysed the role of detrending in the first step of the bootstrap algorithm and also in the bootstrap recursion. He considered OLS, GLS and recursive detrending procedures.

*Address correspondence to: Institute of Applied Economic Studies, Russian Presidential Academy of National Economy and Public Administration, office 2101, build 9, 82, Vernadsky pr., 117571, Moscow, Russia.

E-mail: antonskrobotov@gmail.com 
Another field of research is the development of optimal tests. The seminal paper is Elliott et al. (1996) (hereafter ERS) who developed a nearly (asymptotically) efficient test and obtained Gaussian asymptotic power envelopes for unit root tests. Recently Jansson and Nielsen (2012) developed the likelihood ratio test derived from the full likelihood (in contrast to conditional likelihood, as in standard ADF test, conditioning being with respect to the initial observation). The proposed likelihood ratio test is also nearly (asymptotically) efficient but not asymptotically equivalent to the test proposed by ERS. The likelihood ratio test was further extended to a seasonal unit root context (Jansson and Nielsen (2011)) and cointegration context (Boswijk et al. (2015)).

The standard problem in unit root testing is serious size distortion when the autoregressive or moving average roots are close to -1 . Serious size distortions of Jansson and Nielsen (2012) test are even more pronounced than conventional ADF and $M$ tests in some cases. We propose to use the sieve-based bootstrap version (recoloured bootstrap) in the spirit of Chang and Park (2003) and Cavaliere and Taylor (2009a). Monte-Carlo simulations show the good finite sample properties of the bootstrapped version of Jansson and Nielsen (2012) which outperforms the bootstrap ADF test in some cases.

The paper is organized as follows. In Section 2 we formulate the model and likelihood ratio test statistics. The bootstrap algorithm and its asymptotic properties are discussed in Section 3. The finite sample properties via Monte-Carlo simulations are presented in Section 4. Section 5 concludes. All proofs and additional Monte-Carlo results are contained in the Supplementary Appendix.

\section{Likelihood ratio test}

Consider the following data generating process (DGP):

$$
\begin{aligned}
y_{t} & =\beta^{\prime} d_{t}+u_{t}, t=1, \ldots, T, \\
u_{t} & =\rho u_{t-1}+\varepsilon_{t}, \\
\phi(L) \varepsilon_{t} & =e_{t}
\end{aligned}
$$

where $d_{t}=1$ (constant case) or $d_{t}=(1, t)^{\prime}$ (trend case), $\beta$ is an unknown parameter. The $k$ th order lag polynomial $\phi(z)$ satisfies: (a) $0 \leq p<\infty$, and (b) $\phi(z) \neq 0$ for all $|z| \leq 1$. This assumption is standard and imposes that $\phi(z)$ is a stationary finite-order polynomial, but this assumption can be weakened without changing our main results. The innovation process $e_{t}$ is a martingale difference (with some filtration $\mathcal{F}_{t}$ ) with $E\left(e_{t}^{2} \mid \mathcal{F}_{t-1}\right)=\sigma^{2}$ and $E\left|e_{t}\right|^{r}<K<\infty$ for $r \geq 4$. The initial condition is assumed to be $u_{0}=o_{p}\left(T^{1 / 2}\right)$.

Our purpose is to test the null hypothesis of a unit root, $H_{0}: \rho=1$ against the stationary alternative $H_{1}:|\rho|<1$. Recently Jansson and Nielsen (2012) proposed nearly efficient likelihood ratio unit root test for the testing this null hypothesis $H_{0}$. Let the log likelihood function associated with the model (1)-(3) with $u_{1}=\cdots=u_{-p}=0$ (up to the constant) be ${ }^{1}$

$$
L\left(\rho, \lambda, \beta ; \sigma^{2}, \phi\right)=-\frac{T}{2} \log \sigma^{2}-\frac{1}{2 \sigma^{2}}\left(Y_{\rho, \phi}-D_{\rho_{1}, \rho_{2}, \phi} \beta\right)^{\prime}\left(Y_{\rho, \phi}-D_{\rho, \phi} \beta\right),
$$

where $y_{0}=\cdots=y_{-p}=0, d_{0}=\cdots=d_{-p}=0$, and $p$ is the order of the polynomial $\phi(L)=$ $1-\phi_{1} L-\cdots-\phi_{p} L^{p}$. Matrices $Y_{\rho, \phi}$ and $D_{\rho, \phi}$ are defined as $(1-\rho L) \phi(L) y_{t}$ and $(1-\rho L) \phi(L) d_{t}^{\prime}$,

\footnotetext{
${ }^{1}$ We suppress the dependence of all terms on $T$ for notational convenience.
} 
respectively. Then the likelihood ratio test statistic is written as

$$
\begin{aligned}
L R & =\max _{\bar{\rho} \leq 1, \beta} L\left(\bar{\rho}, \beta ; \hat{\sigma}^{2}, \hat{\phi}^{2}\right)-\max _{\beta} L\left(1,1, \beta ; \hat{\sigma}^{2}, \hat{\phi}^{2}\right) \\
& =\max _{\bar{\rho}} \mathcal{L}\left(\bar{\rho} ; \hat{\sigma}^{2}, \hat{\phi}^{2}\right)-\mathcal{L}\left(1 ; \hat{\sigma}^{2}, \hat{\phi}^{2}\right),
\end{aligned}
$$

where $\hat{\sigma}^{2}$ and $\hat{\phi}$ are the estimators of $\sigma^{2}$ and $\phi=\left(\phi_{1}, \ldots, \phi_{k}\right)^{\prime}$, respectively, and

$$
\mathcal{L}\left(\rho ; \sigma^{2}, \phi^{2}\right)=-\frac{T}{2} \log \sigma^{2}-\frac{1}{2 \sigma^{2}} Y_{\rho, \phi}^{\prime} Y_{\rho, \phi}+\frac{1}{2 \sigma^{2}}\left(Y_{\rho, \phi}^{\prime} D_{\rho, \phi}\right)\left(D_{\rho, \phi}^{\prime} D_{\rho, \phi}\right)^{-1}\left(D_{\rho, \phi}^{\prime} Y_{\rho, \phi}\right)
$$

is the profile $\log$ likelihood function obtained by maximizing $L\left(\rho, \beta ; \sigma^{2}, \phi^{2}\right)$ with respect to the nuisance parameter $\beta$, which is related to the deterministic component. Substituting the consistent estimators $\hat{\sigma}^{2}$ and $\hat{\phi}$ into (5), the $L R$ statistic is maximized with respect to the only one parameter, $\rho$, although there is no closed form expression for this statistic. We discuss the choice of $\hat{\sigma}^{2}$ and $\hat{\phi}$ in Section 4.

Jansson and Nielsen (2012) found the limiting distribution of the $L R^{i}$ test statistic $(i=\mu$ for the constant case and $i=\tau$ for the trend case) under the null hypothesis. In the constant case $\left(d_{t}=1\right)$, we have

$$
L R^{\mu} \Rightarrow \max _{\bar{c} \leq 0} \Lambda(\bar{c})=: \xi^{\mu}
$$

where

$$
\Lambda(\bar{c})=\bar{c} \mathcal{S}-\frac{1}{2} \bar{c}^{2} \mathcal{H}, \mathcal{S}=\frac{1}{2}\left(W(1)^{2}-1\right), \mathcal{H}=\int_{0}^{1} W(r)^{2} d r .
$$

In the trend case $\left(d_{t}=(1, t)^{\prime}\right)$, we have

$$
L R^{\tau} \Rightarrow \max _{\bar{c} \leq 0} \Lambda^{\tau}(\bar{c})=: \xi^{\tau}
$$

where

$$
\Lambda^{\tau}(\bar{c})=\Lambda(\bar{c})+\frac{1}{2} \frac{\left((1-\bar{c}) W(1)+\bar{c}^{2} \int_{0}^{1} r W(r) d r\right)^{2}}{1-\bar{c}+\bar{c}^{2} / 3}-\frac{1}{2} W(1)^{2} .
$$

Remark 1 We note that in the constant case the limiting distribution of the $L R^{\mu}$ test coincides with the case of the absence of the deterministic term. This is a standard result for GLS-based tests.

Remark 2 As Jansson and Nielsen (2012) noted in their Discussion section, the LR test can not be interpreted a an (asymptotically) point optimal test of ERS. This is because the LR test can be expressed as point optimal test with $\hat{c}_{L R}$ as argument, where $\hat{c}_{L R}$ is based on maximising the likelihood function under the alternative hypothesis. However, this estimate $\hat{c}_{L R}$ is random in the limit. Also, while the limiting distribution of the LR test coincides with the limiting distribution of the $A D F-G L S$ test proposed by ERS in the constant case, this is not true for the trend case. This is due to the $A D F-G L S$ test using a plug-in estimator of $\beta$ first based on a fixed non-centrality parameter, say, $\bar{c}_{E R S}$. Then this test profiles out other nuisance parameters $\left(\sigma^{2}\right.$ and $\left.\phi\right)$. The LR test, conversely, uses plug-in estimators of $\sigma^{2}$ and $\phi$, and then profiles out $\beta$ which affect the limiting distribution. Note that these distinctions of the tests are virtually negligible in terms of local asymptotic power. See Jansson and Nielsen (2012, Section 3) for more discussion. 


\section{Bootstrap Likelihood Ratio Test}

As we will see in the simulation section, the likelihood ratio test suffers from serious size distortions when the errors are strongly autocorrelated. This phenomenon is already observed for many other unit root tests (see, e.g., Cavaliere and Taylor (2009a) for so called M unit root tests). The extensive literature proposed to use bootstrap methodology to correct the inference under the null hypothesis. In order to eliminate the influence from serial correlation, in this section we consider sieve based implementation (recoloring) of the bootstrap likelihood ratio unit root test of Jansson and Nielsen (2012). ${ }^{2}$

In this study, we focus on residual based i.i.d. bootstrap of Chang and Park (2003), Park (2002, 2003) with sieve based recoloring. ${ }^{3}$ First we need to define residuals for our botstrap algorithm. Define (quasi) GLS-residuals $\hat{u}_{t}^{i}(i=\mu, \tau)$ as

$$
\hat{u}_{t}^{i}=y_{t}-\hat{\beta}_{i, G L S}^{\prime} d_{t},
$$

where $\hat{\beta}_{i, G L S}^{\prime}$ is the OLS estimate from the regression of $\boldsymbol{y}^{\bar{\rho}}=\left[y_{1},(1-\bar{\rho} L) y_{2}, \ldots,(1-\bar{\rho} L) y_{T}\right]^{\prime}$ on $\boldsymbol{Z}^{\bar{\rho}}=\left[d_{1},(1-\bar{\rho} L) d_{2}, \ldots,(1-\bar{\rho} L) d_{T}\right]^{\prime}$ with $\bar{\rho}=1-\bar{c} / T$ and $\bar{c}=7$ for the constant case and $\bar{c}=13.5$ for the trend case. Next define $\hat{e}_{t}$ as residuals from the fitted ADF regression

$$
\Delta \hat{u}_{t}^{i}=\hat{\rho} \hat{u}_{t-1}^{i}+\sum_{j=1}^{p} \hat{\phi}_{j}^{i} \Delta \hat{u}_{t-j}^{i}+\hat{e}_{t}^{i} .
$$

Next we outline the bootstrap algorithm.

\section{Algorithm 1 (Bootstrap LR Test)}

Step 1: Obtain the standard LR test statistics, $L R^{i}(i=\mu, \tau)$ along with the corresponding OLS residuals, $\hat{e}_{t}^{i}$, from the (quasi) GLS-detrended ADF regression (9). Set $\hat{e}_{t}^{i}=0$ for $t=1, \ldots, p+1$.

Step 2: Generate the vectors of i.i.d. bootstrap errors $\left\{e_{t}^{*}\right\}_{t=1}^{T}$ according to resampling from the centered residuals $\left(\hat{e}_{t}^{i}-\overline{\hat{e}^{i}}\right)$, where $\hat{e}^{i}=T^{-1} \sum_{t=1}^{T} \hat{e}_{t}^{i}$.

Step 3: Construct $u_{t}^{*}$ through the recursion

$$
u_{t}^{*}=\sum_{j=1}^{p} \hat{\phi}_{j} u_{t-j}^{*}+e_{t}^{*}, t=p+1, T,
$$

using estimated parameters $\hat{\phi}_{j}$ from the regression (9) initialized at $u_{1}^{*}=$ $\cdots=u_{p}^{*}=0$ and then construct the bootstrap sample data through the recursion

$$
\Delta y_{t}^{*}=u_{t}^{*}, t=2, \ldots, T
$$

initialized at $y_{1}^{*}=0$.

\footnotetext{
${ }^{2}$ Preliminary simulation showed that the same bootstrap likelihood ratio tests, but without recoloring, do not allow us to fix the size distortions under strongly autocorrelated errors. We do not provide these results to save space.

${ }^{3}$ We also could implement wild bootstrap schemes according Cavaliere and Taylor $(2008,2009 a, b)$ if we suspect a conditionally heteroskedasticity or non-stationary volatility in the errors.
} 
Step 4: Using the bootstrap sample, $\left\{y_{t}^{*}\right\}$, compute the bootstrap LR statistics, denoted $L R^{*, i}(i=\mu, \tau)$ exactly as was done for the original data in Step 1 (including any de-trending), for some fixed lag length $p^{*} \geq 0$.

Step 5: Bootstrap p-values are then defined as: $P_{i, T}^{*}:=G_{i, T}^{*}\left(L R^{i}\right)(i=\mu, \tau)$, where $G_{i, T}^{*}(\cdot)(i=\mu, \tau)$ denote the conditional (on the original sample data) cumulative distribution functions (cdf's) of $L R^{*, i}$. In practice, the cdf's required here will be unknown, but can be approximated in the usual way through numerical simulation.

Remark 3 In Step 1, we use the residuals $\hat{e}_{t}^{i}$ from the (quasi) GLS-detrended ADF regression (9). Smeekes (2012) investigated the effect of the detrending method on asymptotic validity of the bootstrap algorithms. He demonstrated that the bootstrap test was valid for different methods of detrending (recursive or full-time and OLS or GLS) in the first step of the algorithm for generating bootstrap errors. The detrending matters only for constructing bootstrap test statistics due to asymptotic properties of the original asymptotic tests. Moreover, there are other ways to generate the bootstrap sample. For example, we can impose the unit root null hypothesis to obtain $\hat{e}_{t}^{i}$ (see Remark 4 of Cavaliere and Taylor $(2009 a)$ ). But the simulation results seem to be very similar across different methods and we do not report on them. The full set of the simulation results is available upon request.

Remark 4 In Step 4, the lag length $p^{*}$ for the bootstrap tests should not be lower than $k$ from the orignal tests. In our simulations, we just set $p^{*}=p$ as is common in the literature. However, there are studies (see, e.g., Smeekes and Taylor (2012)) where $p^{*}$ is choosen in each bootstrap replication.

We now proceed to the asymptotic properties of our proposed bootstrap algorithm.

Proposition 1 Let the assumptions of (1)-(3) hold. Then $L R^{*, i} \Rightarrow_{p} \xi^{i}(i=\mu, \tau)$, where $\Rightarrow_{p}$ is used to denote weak convergence in probability in the sense of Giné and Zinn (1990). Also, $P_{T}^{*} \stackrel{w}{\rightarrow} U[0,1]$, where $P_{T}^{*}$ is again used generically to denote any of the bootstrap p-values of the LR tests, and $U[0,1]$ denotes a uniform distribution on $[0,1]$.

Remark 5 The Proposition 1 implies that the bootstrap test $L R^{*, i}(i=\mu, \tau)$ attains the same firstorder limiting null distribution as the corresponding original test $L R^{i}$. Moreover, the bootstrap $p$-values are (asymptotically) uniformly distributed under the null hypothesis, implying that the test is asymptotically size controlled.

Remark 6 It can be shown that Proposition 1 also hold under local alternative of the form $\rho=$ $1-c / T$ for $c \geq 0$ and, therefore, the bootstrap LR test has the same local asymptotic power as the local asymptotic power of the size-adjusted original test. Under the fixed alternative, the bootstrap LR test has the same consistency properties as the original LR test.

\section{Finite Sample Size and Power}

In this section we investigate the finite sample behavior of our proposed bootstrap likelihood ratio test. We compare this test with commonly used bootstrap unit root tests proposed by Cavaliere 
and Taylor (2009a) (so-called M-tests) ${ }^{4}$ and Chang and Park (2003) (augmented Dickey-Fuller test), both with autoregressive recolouring.

Our simulations are based on the following DGP (we assume no deterministic component without loss of generality):

$$
\begin{aligned}
& \rho(L) y_{t}=\varepsilon_{t}, \\
& \phi(L) \varepsilon_{t}=\theta(L) e_{t},
\end{aligned}
$$

with $y_{1}=\varepsilon_{1}=0$ and $e_{t} \sim i . i . d . N(0,1)$. The deterministic term is set to be zero without loss of generality. We generate the data for samples of $T=50,100$ and 200 with 10,000 MonteCarlo replications. For analysis of the weak dependence of the errors we focus on (first-order) autoregressive errors $(\phi(L)=1-\phi L, \theta(L)=1)$ and (first-order) moving average errors $(\phi(L)=1$, $\theta(L)=1+\theta L)$. The results are reported for $\phi=\{-0.8,-0.5,0.5,0.8\}$ in the $\operatorname{AR}(1)$ case and $\theta=\{0.8,0.5,-0.5,-0.8\}$ in the MA(1) case. We also report results for the IID case $(\phi(L)=1$, $\theta(L)=1)$.

For implementation of the LR tests we use two different popular types of the consistent estimators of $\sigma^{2}$ and $\phi$. The first is recommended in $(5), \hat{\sigma}^{2}=(T-p-1)^{-1} \sum_{t=k+1}^{T}\left(\Delta y_{t}-\hat{\kappa}^{\prime} Z_{t}\right)^{2}$ and $\hat{\phi}=\left(0, I_{k}\right) \hat{\kappa}$, where $\hat{\kappa}$ is the OLS-estimator in the regression of $\Delta y_{t}$ on $Z_{t}=\left(\Delta d_{t}^{\prime}, \Delta y_{t-1}, \ldots\right.$,

$\left.\Delta y_{t-k}\right)^{\prime}$. These estimators impose the null hypothesis on the regression ADF regression. We denote the LR test with this type of estimators as $L R_{1}^{i}$. The second type uses the ADF-regression under the alternative hypothesis, so that $\hat{\sigma}^{2}=(T-k-1)^{-1} \sum_{t=k+1}^{T}\left(\hat{e}_{t}^{i}\right)^{2}$ and $\hat{\phi}=\left(\hat{\phi}_{1}^{i}, \hat{\phi}_{1}^{i}, \ldots, \hat{\phi}_{k}^{i}\right)^{\prime}$ from the regression (9). We denote the LR test with this type of estimators as $L R_{2}^{i}$.

We compare standard $L R$ tests $\left(L R_{1}\right.$ and $\left.L R_{2}\right)$ with their bootstrap counterparts $\left(L R_{1}^{*}\right.$ and $L R_{2}^{*}$ ) and classical bootstrap tests, $M Z_{\rho}^{*}, M S B^{*}, M Z_{t}^{*}, M P_{t}^{*}$ and $A D F^{*}$. Table 1 represents the size of these tests in the constant case (here $\rho(L)=1-L$ ). For the IID case the size of all tests is close to the nominal one (although slightly undersized). The size of $L R_{1}^{*}$ and $L R_{2}^{*}$ is closer to the nominal one than the other bootstrap tests. For negative $\mathrm{AR}(1)$ errors, $L R_{2}$ is oversized but this oversizing vanishes for its bootstrap counterpart. Again, the $L R_{1}^{*}$ and $L R_{2}^{*}$ control size well while we find serious undersizing for so-called M-tests. For positive AR(1) errors the both $L R_{1}$ and $L R_{2}$ are seriously undersized especially for $\phi=0.8$ even for $T=100$ and 200 . This problem is fixed by applying bootstrap. The size of $L R_{1}^{*}$ and $L R_{2}^{*}$ is closer to the nominal one than all other tests.

Next consider MA(1) errors. For positive $\theta$ we observe greatly improved size for bootstrap LR tests in comparison to their non-bootstrap counterparts. Again, the size is better than other bootstrap tests considered. For negative $\theta$ we have the standard problem of the oversizing of nonbootstrap tests, especially for $L R_{2}$. The size of $L R_{1}^{*}$ and $L R_{2}^{*}$ is considerably smaller than $L R_{1}$ and $L R_{2}$, respectively. However, it is still larger when compared to all other bootstrap tests. Overall, the size of $L R_{1}^{*}$ and $L R_{2}^{*}$ is better for all cases except for a negative MA term. Also, the size improves if the sample size increases for all cases.

Table 2 represents the finite sample power of all tests. We investigate the power of the tests under a near integrated alternative, so that $\rho(L)=1-(1-c / T) L)$ ) with $c=7$ as is common in unit root literature. For $\mathrm{AR}(1)$ errors the power of $L R_{1}^{*}$ and $L R_{2}^{*}$ is the best for all cases except the case of $\phi=-0.8$, where the $A D F^{*}$ test outperforms all other tests. The power of $M$-tests is considerably lower than $L R_{1}^{*}, L R_{2}^{*}$ and $A D F^{*}$. For $M A(1)$ errors, $L R_{1}^{*}$ and $L R_{2}^{*}$ outperform all

\footnotetext{
${ }^{4}$ We note that while Cavaliere and Taylor (2009a) investigate the wild bootstrap with heteroskedastic errors, their results on stationary autoregressive errors dynamic also hold.
} 
other tests when the MA parameter $\theta$ is positive. For negative $\theta$, the $A D F^{*}$ test has greater power. Again, all $M$-tests have lower power than $L R_{1}^{*}, L R_{2}^{*}$ and $A D F^{*}$. Comparing two bootstrap LR tests, $L R_{1}^{*}$ and $L R_{2}^{*}$, we observe very similar power properties except for the case of negative $\theta$. In this case $L R_{2}^{*}$ outperforms $L R_{1}^{*}$. Refering to Table 1 , we observe, that in this case $L R_{2}^{*}$ has a size closer to the nominal one in comparison to $L R_{1}^{*}$.

Tables S.1 and S.2 in Supplementary Appendix show finite sample size and power, respectively, for the trend case (with $c=13.5$ in $\rho(L)=1-(1-c / T) L)$ ). Quantitatively, the results are similar to the constant case, but we have a greater performance of the $L R_{2}^{*}$ test in comparison to $L R_{1}^{*}$ and $A D F^{*}$.

Summarizing results, we found that the $L R_{2}^{*}$ test has great finite sample properties and can be performed in conjunction with the popular bootstrap $A D F$ test. The latter test outperforms the former in the cases of negative MA and very negative AR components across all cases considered.

\section{Conclusion}

In this paper we investigated the bootstrap implementation of the likelihood ratio test for a unit root with different types of nuisance parameter estimators. It was shown that while the standard LR test shows serious size distortion, its bootstrap counterpart has good finite sample properties. We concluded that the bootstrap LR test can be used as a complement for the bootstrap ADF test with GLS detrending.

\section{References}

Basawa, I.V., Mallik, A.K., McCormick, W.P., Reeves, J.H., and Taylor, R.L. (1991a). Bootstrap Test of Significance and Sequential Bootstrap Estimation for Unstable First Order Autoregressive Processes. Communications in Statistics - Theory and Methods, 20, 1015-1026.

Basawa, I.V., Mallik, A.K., McCormick, W.P., Reeves, J.H., and Taylor, R.L. (1991b). Bootstrapping Unstable First-Order Autoregressive Processes. Annals of Statistics, 19, 1098-1101.

Boswijk, P., Jansson, M., and Nielsen, M.Ø. (2015). Improved likelihood ratio tests for cointegration rank in the VAR model. Journal of Econometrics, 184, 97-110.

Cavaliere, G. and Taylor, A. M. R. (2008). Bootstrap unit root tests for time series with nonstationary volatility. Econometric Theory, 24, 43-71.

Cavaliere, G. and Taylor, A. M. R. (2009a). Bootstrap M unit root tests. Econometric Reviews, 28, 393-421.

Cavaliere, G. and Taylor, A. M. R. (2009b). Heteroskedastic time series with a unit root. Econometric Theory, 25, 1228-1276.

Chang, Y. and Park, J. Y. (2003). A sieve bootstrap for the test of a unit root. Journal of Time Series Analysis, 24, 379-400.

Elliott, G., Rothenberg, T.J., and Stock, J.H. (1996). Efficient Tests for an Autoregressive Unit Root. Econometrica, 64, 813-836. 
Giné, E. and Zinn, J. (1990). Bootstrapping general empirical measures. Annals of Probability, 18, 851?-869.

Jansson, M. and Nielsen, M.Ø. (2011). Nearly Efficient Likelihood Ratio Tests for Seasonal Unit Roots. Journal of Tine Series Econometrics, 3, Issue 1.

Jansson, M. and Nielsen, M.Ø. (2012). Nearly Efficient Likelihood Tests of the Unit Root Hypothesis. Econometrica, 80, 2321-2332.

Palm, F.C., Smeekes, S., and Urbain, J.-P. (2008). Bootstrap Unit Root Tests: Comparison and Extensions. Journal of Time Series Analysis, 29, 371-401.

Park, J. Y. (2002). An invariance principle for sieve bootstrap in time series. Econometric Theory, 18, 469-490.

Park, J. Y. (2003). Bootstrap unit root tests. Econometrica, 71, 1845-1895.

Park, J. Y. (2006). A bootstrap theory for weakly integrated processes. Journal of Econometrics, 133, 639-672.

Smeekes, S. (2012). Detrending Bootstrap Unit Root Tests. Econometric Reviews, 32, 869891.

Smeekes, S. and Taylor, A. M. R. (2012). Bootstrap union tests for unit roots in the presence of nonstationary volatility. Econometric Theory, 28, 422-456.

Stock, J.H. (1999). A Class of Tests for Integration and Cointegration, Volume In Cointegration, Causality, and Forecasting: A Festschrift for Clive W.J. Granger. Oxford: Oxford University Press. 
Table 1. Finite sample size (constant case) $-c=0$

\begin{tabular}{|c|c|c|c|c|c|c|c|c|c|c|c|}
\hline & $\phi / \theta$ & $T$ & $L R_{1}$ & $L R_{2}$ & $L R_{1}^{*}$ & $L R_{2}^{*}$ & $M Z_{\rho}^{*}$ & $M Z_{t}^{*}$ & $M S B^{*}$ & $M P_{t}^{*}$ & $A D F^{*}$ \\
\hline \multirow[t]{3}{*}{ IID } & 0 & 50 & 4.0 & 5.5 & 3.6 & 3.6 & 3.3 & 3.3 & 3.4 & 3.3 & 3.5 \\
\hline & & 100 & 3.4 & 4.3 & 3.7 & 3.6 & 3.4 & 3.3 & 3.3 & 3.4 & 3.5 \\
\hline & & 200 & 3.9 & 4.4 & 4.2 & 4.2 & 4.0 & 4.1 & 4.1 & 4.1 & 4.2 \\
\hline \multirow[t]{12}{*}{$\operatorname{AR}(1)$} & -0.8 & 50 & 4.1 & 10.2 & 3.3 & 3.6 & 0.5 & 0.4 & 0.6 & 0.6 & 2.9 \\
\hline & & 100 & 3.6 & 6.2 & 3.1 & 3.0 & 1.5 & 1.4 & 1.7 & 1.7 & 2.9 \\
\hline & & 200 & 4.3 & 5.7 & 3.9 & 3.9 & 3.4 & 3.3 & 3.5 & 3.5 & 4.0 \\
\hline & -0.5 & 50 & 4.0 & 8.8 & 3.7 & 3.8 & 2.1 & 2.2 & 2.2 & 2.2 & 3.2 \\
\hline & & 100 & 4.1 & 6.4 & 3.9 & 3.9 & 3.3 & 3.2 & 3.3 & 3.3 & 3.7 \\
\hline & & 200 & 4.1 & 5.2 & 3.9 & 3.9 & 3.5 & 3.5 & 3.6 & 3.7 & 3.7 \\
\hline & 0.5 & 50 & 0.3 & 2.0 & 3.2 & 3.1 & 1.6 & 1.6 & 1.7 & 1.7 & 2.1 \\
\hline & & 100 & 1.3 & 2.6 & 3.6 & 3.6 & 2.8 & 2.8 & 2.9 & 3.0 & 3.2 \\
\hline & & 200 & 2.4 & 3.3 & 3.8 & 3.9 & 3.5 & 3.5 & 3.6 & 3.7 & 3.9 \\
\hline & 0.8 & 50 & 0.1 & 0.6 & 4.1 & 4.2 & 1.0 & 1.0 & 1.0 & 0.9 & 3.0 \\
\hline & & 100 & 0.3 & 1.0 & 3.8 & 3.7 & 2.8 & 2.8 & 2.6 & 2.6 & 3.3 \\
\hline & & 200 & 1.6 & 2.5 & 4.4 & 4.4 & 4.0 & 4.1 & 4.0 & 4.0 & 4.2 \\
\hline \multirow[t]{12}{*}{$\mathrm{MA}(1)$} & 0.8 & 50 & 0.5 & 3.0 & 3.3 & 3.0 & 0.7 & 0.7 & 0.7 & 0.7 & 1.4 \\
\hline & & 100 & 0.7 & 3.2 & 3.6 & 3.3 & 1.2 & 1.3 & 1.3 & 1.3 & 2.2 \\
\hline & & 200 & 1.4 & 3.9 & 3.9 & 3.6 & 2.3 & 2.4 & 2.4 & 2.5 & 3.5 \\
\hline & 0.5 & 50 & 0.7 & 2.8 & 2.9 & 2.5 & 1.2 & 1.2 & 1.2 & 1.3 & 1.7 \\
\hline & & 100 & 1.3 & 3.3 & 3.3 & 3.2 & 2.5 & 2.5 & 2.5 & 2.5 & 2.9 \\
\hline & & 200 & 2.6 & 4.0 & 4.1 & 3.9 & 3.5 & 3.7 & 3.5 & 3.4 & 3.7 \\
\hline & -0.5 & 50 & 8.5 & 17.8 & 8.9 & 8.6 & 5.6 & 5.7 & 5.6 & 5.5 & 7.4 \\
\hline & & 100 & 6.3 & 11.8 & 6.7 & 6.5 & 4.4 & 4.1 & 4.5 & 4.6 & 5.8 \\
\hline & & 200 & 5.8 & 8.6 & 5.5 & 5.4 & 4.4 & 4.4 & 4.5 & 4.5 & 5.1 \\
\hline & -0.8 & 50 & 26.0 & 47.0 & 28.2 & 27.2 & 16.6 & 16.3 & 16.5 & 16.6 & 23.0 \\
\hline & & 100 & 13.2 & 27.8 & 14.3 & 12.8 & 5.6 & 5.4 & 5.7 & 5.7 & 11.1 \\
\hline & & 200 & 10.5 & 19.9 & 9.9 & 8.4 & 3.4 & 3.4 & 3.4 & 3.5 & 8.2 \\
\hline
\end{tabular}


Table 2. Finite sample power (constant case) $-c=7$

\begin{tabular}{|c|c|c|c|c|c|c|c|c|c|c|c|}
\hline & $\phi / \theta$ & $T$ & $L R_{1}$ & $L R_{2}$ & $L R_{1}^{*}$ & $L R_{2}^{*}$ & $M Z_{\rho}^{*}$ & $M Z_{t}^{*}$ & $M S B^{*}$ & $M P_{t}^{*}$ & $A D F^{*}$ \\
\hline \multirow[t]{3}{*}{ IID } & 0 & 50 & 35.0 & 46.0 & 32.6 & 32.4 & 29.3 & 26.0 & 30.3 & 30.4 & 30.8 \\
\hline & & 100 & 35.7 & 42.7 & 36.9 & 36.7 & 35.0 & 32.4 & 35.5 & 35.7 & 36.5 \\
\hline & & 200 & 39.4 & 43.3 & 40.8 & 40.7 & 39.3 & 37.4 & 40.0 & 40.1 & 40.5 \\
\hline \multirow[t]{12}{*}{$\operatorname{AR}(1)$} & -0.8 & 50 & 26.3 & 57.8 & 22.6 & 28.2 & 4.2 & 3.3 & 4.5 & 4.8 & 25.8 \\
\hline & & 100 & 30.6 & 48.2 & 27.6 & 28.8 & 17.7 & 15.3 & 18.7 & 19.0 & 32.4 \\
\hline & & 200 & 37.8 & 46.7 & 35.3 & 35.4 & 33.1 & 30.7 & 33.9 & 33.9 & 39.1 \\
\hline & -0.5 & 50 & 29.3 & 56.6 & 27.4 & 28.2 & 17.7 & 15.5 & 18.5 & 19.1 & 26.8 \\
\hline & & 100 & 33.1 & 48.4 & 31.4 & 31.7 & 28.9 & 26.9 & 29.5 & 29.6 & 33.2 \\
\hline & & 200 & 39.8 & 48.6 & 38.4 & 38.2 & 37.8 & 35.5 & 38.5 & 38.5 & 40.0 \\
\hline & 0.5 & 50 & 4.5 & 16.0 & 18.1 & 16.7 & 8.0 & 7.0 & 8.6 & 9.7 & 11.8 \\
\hline & & 100 & 17.3 & 29.5 & 33.2 & 32.7 & 27.5 & 25.2 & 28.4 & 28.7 & 30.1 \\
\hline & & 200 & 28.3 & 36.2 & 39.0 & 38.8 & 35.6 & 33.6 & 36.7 & 36.8 & 37.8 \\
\hline & 0.8 & 50 & 0.8 & 7.7 & 20.8 & 20.1 & 6.8 & 6.1 & 7.3 & 8.5 & 14.7 \\
\hline & & 100 & 4.9 & 12.4 & 27.1 & 26.6 & 18.2 & 16.6 & 19.1 & 20.1 & 23.6 \\
\hline & & 200 & 16.4 & 24.1 & 34.6 & 34.4 & 29.3 & 27.2 & 30.3 & 30.9 & 32.8 \\
\hline \multirow[t]{12}{*}{$\mathrm{MA}(1)$} & 0.8 & 50 & 5.3 & 24.7 & 18.6 & 17.3 & 4.9 & 4.4 & 5.2 & 5.9 & 10.6 \\
\hline & & 100 & 8.6 & 29.2 & 26.4 & 25.0 & 13.0 & 12.0 & 13.5 & 14.2 & 20.5 \\
\hline & & 200 & 17.1 & 36.8 & 33.4 & 32.1 & 23.3 & 21.8 & 24.1 & 24.7 & 30.2 \\
\hline & 0.5 & 50 & 9.1 & 24.3 & 17.7 & 16.5 & 8.3 & 7.3 & 9.0 & 9.8 & 11.9 \\
\hline & & 100 & 16.9 & 33.6 & 30.5 & 29.5 & 22.9 & 21.2 & 23.6 & 24.3 & 27.3 \\
\hline & & 200 & 25.6 & 36.9 & 35.4 & 34.6 & 30.4 & 28.5 & 31.4 & 31.9 & 33.9 \\
\hline & -0.5 & 50 & 38.7 & 72.5 & 41.4 & 43.4 & 28.4 & 27.0 & 28.8 & 29.0 & 40.2 \\
\hline & & 100 & 36.7 & 62.1 & 38.8 & 39.1 & 30.3 & 28.9 & 30.7 & 30.7 & 40.1 \\
\hline & & 200 & 43.9 & 59.6 & 43.4 & 43.2 & 39.1 & 37.1 & 39.8 & 39.7 & 45.0 \\
\hline & -0.8 & 50 & 57.0 & 91.7 & 61.7 & 69.7 & 45.9 & 45.4 & 46.1 & 46.3 & 64.3 \\
\hline & & 100 & 40.6 & 83.7 & 45.1 & 52.8 & 26.9 & 26.1 & 27.1 & 27.2 & 55.4 \\
\hline & & 200 & 40.9 & 75.3 & 40.9 & 46.9 & 23.9 & 22.5 & 24.6 & 24.6 & 54.6 \\
\hline
\end{tabular}




\title{
Supplementary Online Appendix \\ to
}

\author{
On Bootstrap Implementation of \\ Likelihood Ratio Test for a Unit Root
}




\section{S.1 Contents}

Section S.1 of this supplement contains mathematical proof of Propositions 1. Section S.2 contains additional Monte Carlo results relating to the trend case.

\section{S.2 Proof of Proposition 1}

As we base our bootstrap sample exactly in the same way as in Smeekes (2012), we have invariance principle for the bootstrap errors $e_{t}^{*}$ :

$$
T^{-1 / 2} \sum_{t=1}^{\lfloor T r\rfloor} e_{t}^{*} \Rightarrow_{p} \sigma W(r),
$$

and the bootstrap sample $y_{t}^{*}$,

$$
T^{-1 / 2} y_{\lfloor T r\rfloor}^{*} \Rightarrow_{p} \sigma \psi(1) W(r),
$$

where $\psi(L)=1+\sum_{j=1}^{\infty} \psi_{j} z^{j}$ is the inverse of $\phi(L)$ (see proof of Theorem 2 of Smeekes (2012), p. 888). Note that Smeekes (2012) considered a weaker assumption about errors (assuming infinitely increasing lag order).

Therefore, as in the proof of Jansson and Nielsen (2012), let $\hat{d}_{t}^{*}=$ $\hat{\phi}^{*}(1)^{-1} \operatorname{diag}(1,1 / \sqrt{T}) \hat{\phi}^{*}(L) d_{t}, \hat{y}_{t}^{*}=\hat{\sigma}^{-1 *} \hat{\phi}^{*}(L) y_{t}^{*}$. Then, the test bootstrap test statistic $L R^{*}=\max _{\bar{c} \leq 0} F\left(\bar{c}, \hat{X}^{*}\right)$, where $\hat{X}^{*}=\left(\hat{S}^{*}, \hat{H}^{*}, \hat{A}^{*}, \hat{B}^{*}\right)$,

$$
\begin{gathered}
\hat{S}^{*}=\hat{\sigma}^{-1 *} T^{-1} \sum_{t=2}^{T} \hat{y}_{t-1}^{*} \Delta \hat{y}_{t}^{*} \Rightarrow_{p} \int_{0}^{1} W(r) d W(r)=: \mathcal{S}, \\
\hat{H}^{*}=\hat{\sigma}^{-2 *} T^{-2} \sum_{t=2}^{T} \hat{y}_{t-1}^{2 *} \Rightarrow_{p} \int_{0}^{1} W(r)^{2} d r=: \mathcal{H} \\
\hat{A}^{*}=\left(\hat{A}^{*}(0), \hat{A}^{*}(1), \hat{A}^{*}(2)\right), \quad \hat{B}^{*}=\left(\hat{B}^{*}(0), \hat{B}^{*}(1), \hat{B}^{*}(2)\right), \\
\hat{A}^{*}(0)=\sum_{t=1}^{T} \Delta \hat{d}_{t} \Delta \hat{y}_{t}^{*} \Rightarrow_{p}\left(\begin{array}{c}
\mathcal{Y} \\
W(1)
\end{array}\right)=: \mathcal{A}(0), \\
\hat{A}^{*}(1)=\sum_{t=1}^{T}\left(\Delta \hat{d}_{t} \hat{y}_{t-1}^{*}+\hat{d}_{t-1} \Delta \hat{y}_{t}^{*}\right) \Rightarrow_{p}\left(\begin{array}{c}
0 \\
W(1)
\end{array}\right)=: \mathcal{A}(1), \\
\hat{A}^{*}(2)=\sum_{t=1}^{T} \hat{d}_{t-1} \hat{y}_{t-1}^{*} \Rightarrow_{p}\left(\begin{array}{cc}
0 \\
\int_{0}^{1} r W(r) d r
\end{array}\right)=: \mathcal{A}(2), \\
\hat{B}^{*}(0)=\sum_{t=1}^{T} \Delta \hat{d}_{t} \Delta \hat{d}_{t}^{\prime *} \Rightarrow_{p}\left(\begin{array}{cc}
K & 0 \\
0 & 1
\end{array}\right)=: \mathcal{B}(0), \\
\hat{B}^{*}(1)=\sum_{t=1}^{T}\left(\Delta \hat{d}_{t} \hat{d}_{t-1}^{\prime *}+\hat{d}_{t-1} \Delta \hat{d}_{t}^{* *}\right) \Rightarrow_{p}\left(\begin{array}{ll}
0 & 0 \\
0 & 1
\end{array}\right)=: \mathcal{B}(1), \\
\hat{B}^{*}(2)=\sum_{t=1}^{T} \hat{d}_{t-1} \hat{d}_{t-1}^{*} \Rightarrow_{p}\left(\begin{array}{cc}
0 & 0 \\
0 & 1 / 3
\end{array}\right)=: \mathcal{B}(2),
\end{gathered}
$$


where limiting results follow from (S.1) and (S.2). As a result, we have $\hat{X}^{*} \Rightarrow_{p} \mathcal{X}=$ $(\mathcal{S}, \mathcal{H}, \mathcal{A}, \mathcal{B})$.

The function $F(\bar{c}, x), x=(s, h, a, b)$ can be written as

$$
\begin{gathered}
F(\bar{c}, x)=\bar{c} s-\frac{1}{2} \bar{c} h+\frac{1}{2} N(\bar{c}, a)^{\prime} D(\bar{c}, b)^{-1} N(\bar{c}, a)-\frac{1}{2} N(0, a)^{\prime} D(0, b)^{-1} N(0, a), \\
N(\bar{c}, a)=a(0)-\bar{c} a(1)+\bar{c}^{2} a(2), \quad D(\bar{c}, b)=b(0)-\bar{c} b(1)+\bar{c}^{2} b(2),
\end{gathered}
$$

therefore $F\left(\bar{c}, \hat{X}^{*}\right) \Rightarrow_{p} F(\bar{c}, \mathcal{X})=\Lambda^{\tau}(\bar{c})$ for every $\bar{c} \leq 0$. The convergence result $L R^{*, \tau}=$ $\max _{\bar{c} \leq 0} F\left(\bar{c}, \hat{X}^{*}\right) \Rightarrow_{p} \max _{\bar{c} \leq 0} F(\bar{c}, \mathcal{X})$ follows from the same lines as in the proof in Jansson and Nielsen (2012).

\section{S.3 Additional Monte Carlo Results}

This section contains additional Monte Carlo results relating to the trend case. Tables S.1-S.2 give complementary results to those given in Tables $1-2$ respectively. The Monte Carlo DGP and set-up of these experiments were otherwise exactly as detailed in Section 4. 
Table S.1. Finite sample size (trend case) $-c=0$

\begin{tabular}{|c|c|c|c|c|c|c|c|c|c|c|c|}
\hline & $\phi / \theta$ & $T$ & $L R_{1}$ & $L R_{2}$ & $L R_{1}^{*}$ & $L R_{2}^{*}$ & $M Z_{\rho}^{*}$ & $M Z_{t}^{*}$ & $M S B^{*}$ & $M P_{t}^{*}$ & $A D F^{*}$ \\
\hline \multirow[t]{3}{*}{ IID } & 0 & 50 & 2.8 & 6.8 & 3.3 & 3.3 & 3.4 & 3.6 & 3.3 & 3.4 & 3.1 \\
\hline & & 100 & 3.0 & 5.1 & 3.3 & 3.3 & 3.2 & 3.3 & 3.2 & 3.2 & 3.2 \\
\hline & & 200 & 3.2 & 4.2 & 3.4 & 3.3 & 3.4 & 3.4 & 3.4 & 3.4 & 3.4 \\
\hline \multirow[t]{12}{*}{$\operatorname{AR}(1)$} & -0.8 & 50 & 2.8 & 21.6 & 3.8 & 6.0 & 0.1 & 0.1 & 0.2 & 0.2 & 2.5 \\
\hline & & 100 & 2.7 & 10.2 & 2.9 & 3.4 & 0.4 & 0.4 & 0.4 & 0.4 & 2.6 \\
\hline & & 200 & 3.2 & 7.0 & 3.2 & 3.2 & 1.6 & 1.4 & 1.6 & 1.7 & 3.1 \\
\hline & -0.5 & 50 & 3.1 & 17.2 & 4.9 & 5.5 & 1.8 & 1.7 & 1.8 & 1.8 & 3.7 \\
\hline & & 100 & 3.1 & 9.5 & 3.6 & 3.6 & 2.2 & 2.2 & 2.2 & 2.2 & 3.2 \\
\hline & & 200 & 3.2 & 6.0 & 3.3 & 3.3 & 3.1 & 3.1 & 3.0 & 3.0 & 3.3 \\
\hline & 0.5 & 50 & 0.0 & 0.6 & 1.6 & 1.3 & 0.6 & 0.7 & 0.5 & 0.4 & 0.7 \\
\hline & & 100 & 0.4 & 3.0 & 3.3 & 3.4 & 2.8 & 2.9 & 2.7 & 2.6 & 3.0 \\
\hline & & 200 & 1.4 & 3.2 & 3.4 & 3.4 & 3.2 & 3.2 & 3.1 & 3.2 & 3.2 \\
\hline & 0.8 & 50 & 0.0 & 0.2 & 1.9 & 1.7 & 1.0 & 1.2 & 0.9 & 0.7 & 2.7 \\
\hline & & 100 & 0.0 & 0.4 & 3.0 & 3.0 & 2.6 & 2.7 & 2.4 & 2.4 & 3.3 \\
\hline & & 200 & 0.3 & 1.1 & 3.7 & 3.7 & 3.5 & 3.5 & 3.5 & 3.4 & 3.5 \\
\hline \multirow[t]{12}{*}{$\mathrm{MA}(1)$} & 0.8 & 50 & 0.1 & 2.5 & 2.0 & 2.1 & 0.3 & 0.3 & 0.3 & 0.2 & 0.5 \\
\hline & & 100 & 0.2 & 3.8 & 2.7 & 2.9 & 0.6 & 0.6 & 0.6 & 0.7 & 1.1 \\
\hline & & 200 & 0.3 & 4.5 & 3.1 & 2.8 & 1.1 & 1.1 & 1.1 & 1.1 & 1.6 \\
\hline & 0.5 & 50 & 0.2 & 1.9 & 1.2 & 1.2 & 0.4 & 0.5 & 0.3 & 0.3 & 0.4 \\
\hline & & 100 & 0.4 & 3.9 & 2.8 & 2.8 & 1.6 & 1.7 & 1.7 & 1.7 & 2.1 \\
\hline & & 200 & 1.1 & 3.8 & 2.7 & 2.6 & 2.2 & 2.2 & 2.2 & 2.2 & 2.3 \\
\hline & -0.5 & 50 & 11.2 & 35.7 & 15.3 & 16.5 & 9.5 & 9.5 & 9.6 & 9.5 & 12.5 \\
\hline & & 100 & 5.9 & 20.9 & 8.2 & 8.2 & 4.5 & 4.5 & 4.5 & 4.5 & 6.2 \\
\hline & & 200 & 4.3 & 12.4 & 5.5 & 5.2 & 3.7 & 3.6 & 3.7 & 3.8 & 4.9 \\
\hline & -0.8 & 50 & 36.1 & 76.4 & 44.7 & 48.2 & 28.8 & 28.7 & 28.9 & 28.9 & 37.6 \\
\hline & & 100 & 19.0 & 55.7 & 24.9 & 25.5 & 11.5 & 11.4 & 11.5 & 11.6 & 19.4 \\
\hline & & 200 & 9.1 & 35.0 & 12.8 & 11.5 & 3.0 & 2.9 & 3.1 & 3.1 & 9.0 \\
\hline
\end{tabular}


Table S.2. Finite sample power (trend case) $-c=13.5$

\begin{tabular}{|c|c|c|c|c|c|c|c|c|c|c|c|}
\hline & $\phi / \theta$ & $T$ & $L R_{1}$ & $L R_{2}$ & $L R_{1}^{*}$ & $L R_{2}^{*}$ & $M Z_{\rho}^{*}$ & $M Z_{t}^{*}$ & $M S B^{*}$ & $M P_{t}^{*}$ & $A D F^{*}$ \\
\hline \multirow[t]{3}{*}{ IID } & 0 & 50 & 30.8 & 56.8 & 35.1 & 35.4 & 33.6 & 33.1 & 33.6 & 33.7 & 32.7 \\
\hline & & 100 & 33.6 & 48.5 & 36.2 & 36.2 & 34.8 & 34.2 & 35.2 & 35.3 & 35.1 \\
\hline & & 200 & 36.3 & 46.2 & 38.3 & 38.2 & 36.9 & 36.5 & 37.3 & 37.5 & 37.6 \\
\hline \multirow[t]{12}{*}{$\operatorname{AR}(1)$} & -0.8 & 50 & 22.3 & 82.8 & 29.8 & 45.2 & 0.8 & 0.7 & 0.8 & 0.9 & 24.9 \\
\hline & & 100 & 26.6 & 64.9 & 29.0 & 33.9 & 4.7 & 4.4 & 4.9 & 4.9 & 29.4 \\
\hline & & 200 & 32.5 & 54.1 & 33.0 & 34.0 & 19.6 & 19.0 & 20.1 & 20.2 & 34.1 \\
\hline & -0.5 & 50 & 23.7 & 76.1 & 34.3 & 39.7 & 11.9 & 11.6 & 12.3 & 12.4 & 28.1 \\
\hline & & 100 & 27.0 & 60.1 & 31.0 & 32.1 & 22.1 & 21.3 & 22.7 & 22.9 & 30.1 \\
\hline & & 200 & 33.9 & 53.4 & 36.0 & 36.1 & 32.4 & 31.8 & 32.7 & 32.8 & 35.5 \\
\hline & 0.5 & 50 & 0.6 & 4.1 & 2.8 & 2.5 & 1.1 & 1.2 & 1.2 & 1.2 & 1.4 \\
\hline & & 100 & 4.2 & 23.2 & 21.9 & 21.5 & 17.0 & 16.8 & 17.0 & 17.0 & 18.2 \\
\hline & & 200 & 18.8 & 34.6 & 33.4 & 33.2 & 30.3 & 29.8 & 30.6 & 31.0 & 31.9 \\
\hline & 0.8 & 50 & 0.0 & 1.8 & 7.0 & 6.5 & 2.1 & 2.1 & 2.0 & 1.8 & 4.6 \\
\hline & & 100 & 0.4 & 5.8 & 16.2 & 16.4 & 11.6 & 11.7 & 11.7 & 11.7 & 14.2 \\
\hline & & 200 & 3.9 & 12.3 & 23.2 & 23.3 & 20.2 & 19.8 & 20.4 & 20.8 & 22.1 \\
\hline \multirow[t]{12}{*}{$\mathrm{MA}(1)$} & 0.8 & 50 & 1.3 & 18.2 & 6.6 & 7.4 & 1.8 & 1.6 & 1.8 & 1.9 & 2.2 \\
\hline & & 100 & 3.0 & 30.1 & 16.1 & 17.5 & 6.7 & 6.5 & 6.8 & 7.0 & 10.3 \\
\hline & & 200 & 6.3 & 37.2 & 22.8 & 23.1 & 12.8 & 12.6 & 13.0 & 13.1 & 17.5 \\
\hline & 0.5 & 50 & 3.8 & 15.5 & 6.1 & 6.0 & 4.5 & 4.2 & 4.7 & 4.7 & 4.2 \\
\hline & & 100 & 6.4 & 32.6 & 20.2 & 20.1 & 13.3 & 13.0 & 13.6 & 13.5 & 15.8 \\
\hline & & 200 & 15.5 & 37.2 & 27.7 & 27.2 & 23.5 & 23.1 & 23.8 & 23.9 & 25.5 \\
\hline & -0.5 & 50 & 43.2 & 89.9 & 54.9 & 61.6 & 37.1 & 36.8 & 37.1 & 37.3 & 48.5 \\
\hline & & 100 & 31.9 & 77.2 & 42.4 & 44.2 & 26.4 & 25.8 & 26.4 & 26.5 & 38.3 \\
\hline & & 200 & 33.4 & 67.9 & 40.4 & 40.1 & 29.9 & 29.3 & 30.2 & 30.4 & 38.9 \\
\hline & -0.8 & 50 & 75.2 & 98.9 & 82.1 & 89.9 & 68.2 & 68.0 & 68.2 & 68.2 & 78.9 \\
\hline & & 100 & 53.8 & 97.0 & 63.0 & 73.4 & 43.5 & 43.4 & 43.5 & 43.4 & 62.4 \\
\hline & & 200 & 37.7 & 91.5 & 49.0 & 55.2 & 21.6 & 21.2 & 21.9 & 21.9 & 49.8 \\
\hline
\end{tabular}

